\title{
A New Class of Robust Feedback Controllers for Dynamical Systems in the Absence of Matching Conditions
}

\author{
Hansheng $\mathrm{WU}^{*}$ and Koichi MizUKAMI*
}

\begin{abstract}
In this paper we consider a class of dynamical systems with unmatched uncertainties. We propose a new class of state feedback controllers which can guarantee global exponential stability of dynamical systems with unmatched uncertainties as long as a bounding function on perturbations of the uncertainties is available. The state feedback controller developed here is continuous and bounded, and can guarantee faster convergence rate and better transient behavior. A numerical example is given to demonstrate the utilization of our approach.
\end{abstract}

Key Words : robust stabilization, state feedback controller synthesis, exponential stability, uncertainty, Lyapunov function, matching condition

\section{Introduction}

It is well known that robust control has been one of the major research subjects in the area of control systems due to the existence of uncertainty in a large number of practical control systems, and many solution approaches have been developed ${ }^{1) \sim 10}$. In Ref. 1), for example, asymptotic stability of uncertain dynamical systems is investigated by using Lyapunov minmax approach. In Ref. 2), based on the stability of a nominal system (i.e. the system in the absence of uncertainty), a class of continuous state feedback controllers guaranteeing uniform ultimate boundedness for uncertain dynamical systems are proposed. In Ref. 3), Lyapunov-function theory is used to design a class of stabilizing controllers for uncertain dynamical systems including linear and nonlinear, and the concept of the practical stability is presented. In Ref. 4), by using Lyapunov methods for robust controller design in conjunction with differential geometric methods, a class of stabilizing controllers for uncertain nonlinear dynamical system are synthesized. In Ref. 5), a control law guaranteeing practical stability of uncertain dynamical systems is developed, when

\footnotetext{
* Faculty of Integrated Arts and Sciences, Hiroshima University, 1-7-1 Kagamiyama, Higashi-Hiroshima

(Received June 17, 1993)

(Revised October 4, 1993)
}

the input matrix of an affine system is not invertible but posi-invertible for some positive definite function. Actually, the design of stabilizing controllers in Refs. 1) 5) is based on Lyapunov min-max approach.

Generally, by making use of Lyapunov min-max approach, the assumptions about uncertainties, called the matching conditions, are often required. Therefore, there are some papers that devote to relax these matching assumptions. In Refs. 7) 9), for example, robust stability of uncertain dynamical systems are discussed by introducing the notions of measure of mismatching threshold. But, in these papers, the unmatched uncertainties are required to be smaller than the threshold value so that a stability result holds only locally with respect to the size of the uncertainties. In Ref. 10), matching assumptions are further relaxed by introducing the concept of equivalently matched uncertainties.

In this paper, we consider a class of dynamical systems with unmatched uncertainties. We propose a new class of robust state feedback controllers such that globally exponential stability of uncertain dynamical systems can be guaranteed in the absence of matching assumptions as long as a bounding function on uncertainties is available. By making use of the approach developed here, we can also obtain an estimate inequality on the rate of convergence. The state feedback controller proposed in this paper is 
continuous and bounded, and can guarantee faster convergence rate and better transient behavior. A numerical example is given to demonstrate the utilization of our results.

This paper consists of the following parts. In Section 2, the problem to be tackled in this paper is precisely stated, and some necessary assumptions are introduced. In Section 3, we propose a class of stabilizing state feedback controllers, and discuss some relative problems. In Section 4, a numerical example in which uncertain dynamical system does not satisfy the so called matching conditions is given to demonstrate the utilization of our results. Finally, the paper is concluded in Section 5 with a brief discussion of the results.

\section{Problem Formulation and Assumptions}

\section{1 Problem formulation}

Consider uncertain dynamical systems described by the following state equations.

$$
\begin{aligned}
& \frac{d x(t)}{d t}=A x(t)+B u(t)+\xi(x, t) \\
& x\left(t_{0}\right)=x^{0}
\end{aligned}
$$

where $t \in R$ is time, $x(t) \in R^{n}$ is the state vector, $u(t)$ $\in R^{m}$ is the control vector, $A \in R^{n \times n}$ and $B \in R^{n \times m}$ are constant matrices, and $\xi(x, t): R^{n} \times R \rightarrow R^{n}$ denotes the system uncertainties. The uncertain $\xi(x, t)$ is unmatched and is assumed to be bounded in magnitude, usually in its Euclidean norm denoted by $\|\cdot\|$. In addition, we also assume that $\xi(0, t)=0$. The corresponding system without uncertainty, called nominal system, is described by

$$
\begin{aligned}
& \frac{d x(t)}{d t}=A x(t)+B u(t) \\
& x\left(t_{0}\right)=x^{0}
\end{aligned}
$$

Here, without loss of any generality, nominal system (2) is assumed to be stabilizable. It is based on such an assumption that we can discuss the robust stability of uncertain dynamical system (1).

Now, the question is to synthesize a robust state feedback controller $u(t)$ such that system (1) is stable in the presence of the uncertain $\xi(x, t)$.

Remark 1: It is well known that if the uncertain $\xi(x, t)$ is matched (i. e. there is an unknown function $\xi^{\prime}(x, t)$ such that $\left.\xi(x, t)=B \xi^{\prime}(x, t)\right)$ and if the bounding function on the size of uncertainties $\xi^{\prime}(x, t)$ is known, there exist a few feedback controllers (e.g. see Refs. 1), 2), 6)) that yield certain types of stability for system (1). But, these results on global stability hold in general only if $\xi(x, t)$ is matched. In this paper, we want to develop a new approach to synthesizing a class of state feedback controllers which can guarantee a global stability result even if the uncertain $\xi(x, t)$ is unmatched.

\subsection{Assumptions}

Before giving the synthesis approach, we introduce for dynamical system (1) the following standard assumptions.

Assumption 1: The uncertain $\xi(.,):. R^{n} \times R \rightarrow R^{n}$ is bounded in Euclidean norm by a known function. That is, there is a known nonnegative, continuous function $\rho(.,):. R^{n} \times R \rightarrow R^{+}$such that

$$
\|\xi(x, t)\| \leq \rho(x, t)
$$

for all $(x, t) \in R^{n} \times R$.

In addition, the function $\rho(.,$.$) is assumed without$ loss of any generality to be uniformly bounded with respect to time and locally uniformly bounded with respect to the state $x$.

Assumption 2: The matrix pair $(A, B)$ defined in dynamical system ( 1 ) is completely controllable.

It follows from Assumption 2 that for any symmetric positive definite matrix $Q \in R^{n \times n}$ and any positive constant $\alpha$, the algebraic Riccati equation of the form

$$
(A+\alpha I)^{T} P+P(A+\alpha I)-P B B^{T} P=-Q
$$

has a solution $P \in R^{n \times n}$, which is also a symmetric positive definite matrix.

Assumption 3 : Letting $M$ be a set defined by

$$
M:=\left\{(x, t):\left\|B^{T} P x\right\|=0\right\}
$$

then, we assume that for any $(x, t) \in M$,

$$
\rho(x, t)<\alpha \cdot \frac{\sqrt{\lambda_{\min }(P)}}{\lambda_{\max }(P)} \sqrt{V(x)}
$$

where $V(x)=x^{T} P x$.

\section{Main Results}

For dynamical system ( 1 ) with unmatched uncertain $\xi(x, t)$, we propose the following state feedback controller.

$$
u(t)=u_{1}(t)+u_{2}(t)
$$

where

$$
u_{1}(t)=-\frac{1}{2} B^{T} P x
$$




$$
\begin{aligned}
u_{2}(t)= & -\frac{\lambda_{\max }(P)}{\sqrt{\lambda_{\min }(P)}} \\
& \cdot \frac{\delta(x, t) B^{T} P x \rho^{2}(x, t)}{\sqrt{x^{T} P x} \rho(x, t)+\varepsilon\|x\| \exp \left\{-\beta\left(t-t_{0}\right)\right\}}
\end{aligned}
$$

where $\varepsilon$ and $\beta$ are positive constants. In this paper, $\lambda_{\max }(\cdot)$ and $\lambda_{\min }(\cdot)$ denote the maximum and minimum eigenvalues of the matrix $(\cdot)$, respectively. Without loss of any generality, we select that $\alpha \neq \beta$. In addition, $\delta(\cdot)$ is selected such that

$$
\delta(x, t)=\frac{x^{T} P x}{\left\|B^{T} P x\right\|^{2}}, \quad(x, t) \notin M
$$

It is apparent that the state feedback controller (6) with ( 7 ) is continuous and bounded.

Thus, from the controller ( 6 ), we have the following theorem which shows globally exponential stability of dynamical system ( 1 ).

Theorem : Consider dynamical system ( 1 ) satisfying Assumption 1-3. Then, the closed-loop dynamical system ( 1 ), ( 6 ) with ( 7 ) is globally exponentially stable. More precisely, we have

$$
\begin{aligned}
& \|x(t)\| \leq \sigma^{\frac{1}{2}}\left\|x^{0}\right\| \exp \left\{-\alpha\left(t-t_{0}\right)\right\} \\
& +\frac{\varepsilon \sigma}{(\alpha-\beta) \sqrt{\lambda_{\min }(P)}}\left[\exp \left\{-\beta\left(t-t_{0}\right)\right\}\right. \\
& \left.-\exp \left\{-\alpha\left(t-t_{0}\right)\right\}\right]
\end{aligned}
$$

where $\sigma=\lambda_{\max }(P) / \lambda_{\min }(P)$.

Proof: Applying the state feedback controller ( 6 ) with (7.a) to (1) yields

$$
\frac{d x}{d t}=\left(A-\frac{1}{2} B B^{T} P\right) x+B u_{2}+\xi(x, t)
$$

We first define a scalar function as follows.

$$
V(x)=x^{T} P x
$$

where $P$ is the solution of the algebraic Riccati equation (4).

By the Rayleigh's principle, we have

$$
\lambda_{\min }(P)\|x\|^{2} \leq V(x) \leq \lambda_{\max }(P)\|x\|^{2}
$$

It follows from (4) and (10) that

$$
\begin{aligned}
\frac{d V(x)}{d t}= & -2 \alpha x^{T} P x-x^{T} Q x+2 x^{T} P \xi(x, t) \\
& +2 x^{T} P B u_{2} \\
\leq & -2 \alpha V(x)+2\|P x\| \rho(x, t)+2 x^{T} P B u_{2}
\end{aligned}
$$

Since $P$ is a symmetric positive matrix, from (11) we have

$$
\|P x\| \leq \lambda_{\max }(P)\|x\| \leq \frac{\lambda_{\max }(P)}{\sqrt{\lambda_{\min }(P)}} \sqrt{x^{T} P x}
$$

By making use of (7.b) and (13), from (12) we have

$$
\begin{aligned}
\frac{d V(x)}{d t} \leq & -2 \alpha V(x) \\
& +\frac{2 \lambda_{\max }(P)}{\sqrt{\lambda_{\min }(P)}}\left[\sqrt{x^{T} P x} \cdot \rho(x, t)\right. \\
& \left.-\frac{\delta(x, t)\left\|B^{T} P x\right\|^{2} \cdot \rho^{2}(x, t)}{\sqrt{x^{T} P x} \cdot \rho(x, t)+\varepsilon\|x\| \exp \left\{-\beta\left(t-t_{0}\right)\right\}}\right]
\end{aligned}
$$

For $(x, t) \in M$, it is obvious from (14) and Assumption 3 that

$$
\begin{gathered}
\frac{d V(x)}{d t} \leq- \\
\cdot \rho(x, t)<0
\end{gathered}
$$

That is, when $(x, t) \in M$, system ( 1$)$ is stable.

Furthermore, for $(x, t) \notin M$, from ( 8 ) and (14) we obtain

$$
\begin{gathered}
\frac{d V(x)}{d t} \leq-2 \alpha V(x)+\frac{2 \lambda_{\max }(P)}{\sqrt{\lambda_{\min }(P)}}\left[\sqrt{x^{T} P x} \cdot \rho(x, t)\right. \\
\left.-\frac{x^{T} P x \cdot \rho^{2}(x, t)}{\sqrt{x^{T} P x} \cdot \rho(x, t)+\varepsilon\|x\| \exp \left\{-\beta\left(t-t_{0}\right)\right\}}\right] \\
=-2 \alpha V(x)+\frac{2 \lambda_{\max }(P)}{\sqrt{\lambda_{\min }(P)}} \\
\cdot \frac{\sqrt{x^{T} P x} \cdot \rho(x, t) \cdot \varepsilon\|x\| \exp \left\{-\beta\left(t-t_{0}\right)\right\}}{\sqrt{x^{T} P x} \cdot \rho(x, t)+\varepsilon\|x\| \exp \left\{-\beta\left(t-t_{0}\right)\right\}}
\end{gathered}
$$

Therefore, it follows from (15) and from the inequality

$$
0 \leq \frac{a b}{a+b} \leq b, \forall a, b \geq 0
$$

that

$$
\begin{aligned}
\frac{d V(x)}{d t} \leq & -2 \alpha V(x) \\
& +\frac{2 \varepsilon \lambda_{\max }(P)}{\sqrt{\lambda_{\min }(P)}}\|x\| \exp \left\{-\beta\left(t-t_{0}\right)\right\}
\end{aligned}
$$

From (16) and (11), we can obtain an inequlaity on $V(x)$ as follows.

$$
\begin{aligned}
V(x) \leq & \lambda_{\max }(P)\left\|x^{0}\right\|^{2} \exp \left\{-2 \alpha\left(t-t_{0}\right)\right\} \\
& +\int_{t_{0}}^{t} \frac{2 \varepsilon \lambda_{\max }(P)}{\sqrt{\lambda_{\min }(P)}} \exp \{-2 \alpha(t-\tau)\}\|x(\tau)\| \\
& \exp \left\{-\beta\left(\tau-t_{0}\right)\right\} d \tau
\end{aligned}
$$

Here, we define an auxiliary function $S(t)$ as follows.

$$
\begin{aligned}
S(t)= & {\left[\lambda_{\max }(P)\left\|x^{0}\right\|^{2} \exp \left\{-2 \alpha\left(t-t_{0}\right)\right\}\right.} \\
& +\int_{t_{0}}^{t} \frac{2 \varepsilon \lambda_{\max }(P)}{\sqrt{\lambda_{\min }(P)}} \exp \{-2 \alpha(t-\tau)\}\|x(\tau)\| \\
& \left.\exp \left\{-\beta\left(\tau-t_{0}\right)\right\} d \tau\right]^{\frac{1}{2}}
\end{aligned}
$$

Then, comparing (17) with (18) we have

$$
S(t) \geq \sqrt{V(x)} \geq \sqrt{\lambda_{\min }(P)}\|x(t)\|
$$

It follows from (19) that 


$$
\frac{\|x(t)\|}{S(t)} \leq \frac{1}{\sqrt{\lambda_{\min }(P)}}
$$

On the other hand, differentiating (18) yields

$$
\begin{aligned}
\frac{d S(t)}{d t}= & -\alpha S(t)+\varepsilon \cdot \frac{\lambda_{\max }(P)}{\sqrt{\lambda_{\min }(P)}} \cdot \frac{\|x(t)\|}{S(t)} \\
& \cdot \exp \left\{-\beta\left(t-t_{0}\right)\right\} \\
\leq & -\alpha S(t)+\varepsilon \sigma \exp \left\{-\beta\left(t-t_{0}\right)\right\}
\end{aligned}
$$

Moreover, from (21) we obtain an inequality on $S(t)$ as follows.

$$
\begin{aligned}
S(t) \leq & \sqrt{\lambda_{\max }(P)}\left\|x^{0}\right\| \exp \left\{-\alpha\left(t-t_{0}\right)\right\} \\
& +\varepsilon \sigma \int_{t_{0}}^{t} \exp \{-\alpha(t-\tau)\} \exp \left\{-\beta\left(\tau-t_{0}\right)\right\} d \tau \\
= & \sqrt{\lambda_{\max }(P)}\left\|x^{0}\right\| \exp \left\{-\alpha\left(t-t_{0}\right)\right\} \\
& +\frac{\varepsilon \sigma}{\alpha-\beta}\left[\exp \left\{-\beta\left(t-t_{0}\right)\right\}-\exp \left\{-\alpha\left(t-t_{0}\right)\right\}\right]
\end{aligned}
$$

From (19) and (22), we can easily obtain (9). Therefore, it is shown from (9) that closed-loop dynamical system (1), (6) with (7) is globally exponentially stable in the presence of completely unmatched uncertainties.

Remark 2: Our approach can easily extended to uncertain nonlinear dynamical systems in the absence of matching conditions. In fact, based on the stability of a nominal system (i.e. there exists a Lyapunov function for nominal nonlinear system), by the method similar to that of this paper, we can synthesize a class of robust state feedback controllers for uncertain nonlinear dynamical systems.

Remark 3: In the proof of Theorem, we have discussed the stability of uncertain closed-loop dynamical system ( 1 ), (6) by dividing the inequality (14) into two cases, i. e. $(x, t) \in M$ and $(x, t) \notin M$, respectively. It is obvious from (14) that for any ( $x$, $t) \in M$, the state feedback controller (7.b) is equal to zero. This implies that for any $(x, t) \in M$, the controller (7.b) can not compensate the perturbations of the uncertain $\xi(x, t)$. Therefore, for this cases, it is necessary to introduce some assumptions on the bounding function of uncertainties (e. g. see Assumption 3 in this paper).

Remark 4: In the light of the proof of Theorem, a procedure for synthesizing a stabilizing state feedback controller may be suggested as follows. (i) Firstly, selecting an appropriate symmetric positive definite matrix $Q$ and a positive constant $\alpha$, from (4) we can obtain a symmetric positive definite matrix $P$. (ii) Secondly, for any $(x, t) \in M$, we must check whether the condition (5) is satisfied or not. (iii) Thirdly, we choose appropriate positive constants $\varepsilon$ and $\beta$. Then, from ( 6 ) and (7), we can obtain a stabilizing state feedback controller. Such a synthesis procedure will further be illustrated by a numerical example in the next section.

\section{Illustrative Example}

In the last section, we have proposed a new class of state feedback controllers which can guarantee globally exponential stability of dynamical systems with completely unmatched uncertainties. In this section, we give a numerical example to demonstrate the utilization of our results.

Consider a dynamical system with completely unmatched uncertainties described by the following state equation.

$$
\frac{d x(t)}{d t}=\left[\begin{array}{ll}
0 & 2 \\
2 & 1
\end{array}\right] x(t)+\left[\begin{array}{ll}
0 & 1 \\
0 & 1
\end{array}\right] u(t)+\xi(x, t)
$$

where we assume that the uncertain $\xi(x, t)$ satisfies

$$
\|\xi(x, t)\| \leq \rho(x, t)=\sqrt{0.25 x_{1}^{2}+0.64\left|x_{1} x_{2}\right|}
$$

Here, we will develop a state feedback controller such that globally expential stability of dynamical system (23) can be guaranteed in the presence of the uncertain perturbation (24). In the light of Section 3 , the solution may be given as follows.

First, we select a symmetric positive definite matix $Q$ as follows.

$$
Q=\left[\begin{array}{ll}
4.5 & 1.5 \\
1.5 & 0.6
\end{array}\right]
$$

Then, from (4) we have that for a positive constant $\alpha=2$,

$$
P=\left[\begin{array}{rr}
8.03 & -4.25 \\
-4.25 & 10.51
\end{array}\right]
$$

Furthermore, from (25) we also have

$$
\lambda_{\min }(P)=4.84, \quad \lambda_{\max }(P)=13.69
$$

Secondly, we check whether the condition $(5)$ is satisfied or not. For any $(x, t) \in M:=\left\{(x, t) \in R^{2} \times\right.$ $\left.R:\left\|B^{T} P x\right\|=\left|3.78 x_{1}+6.26 x_{2}\right|=0\right\}$, we have

$$
\rho(x, t)=0.789\left|x_{1}(t)\right|
$$

On the other hand, for any $(x, t) \in M$, we also have

$$
\alpha \frac{\sqrt{\lambda_{\min }(P)}}{\lambda_{\max }(P)} \sqrt{V(x)} \geq \alpha \frac{\lambda_{\min }(P)}{\lambda_{\max }(P)}\|x(t)\|=0.895\left|x_{1}(t)\right|
$$

Then, it follows from (27) and (28) that for any ( $x$, 
$t) \in M$,

$$
\rho(x, t) \leq \alpha \frac{\sqrt{\lambda_{\min }(P)}}{\lambda_{\max }(P)} \sqrt{V(x)}
$$

That is, Assumption 3 is satisfied for any $(x, t) \in M$.

Thirdly, we choose positive constants $\varepsilon$ and $\beta$ as follows.

$$
\varepsilon=2.0, \quad \beta=3.0
$$

Therefore, from (25), (26), and (29) we have a state feedback controller as follows.

$$
u(t)=u_{1}(t)+u_{2}(t)
$$

where

$$
\begin{aligned}
& u_{1}(t)=-\left[\begin{array}{cc}
0 & 0 \\
1.89 & 3.13
\end{array}\right] x(t) \\
& u_{2}(t)=-\rho_{c}(x, t)\left[\begin{array}{cc}
0 & 0 \\
23.52 & 38.95
\end{array}\right] x(t)
\end{aligned}
$$

where

$$
\rho_{c}(x, t)=\frac{\delta(x, t) \rho^{2}(x, t)}{\sqrt{x^{T} P x} \rho(x, t)+2.0\|x\| \exp \left\{-3.0\left(t-t_{0}\right)\right\}}
$$

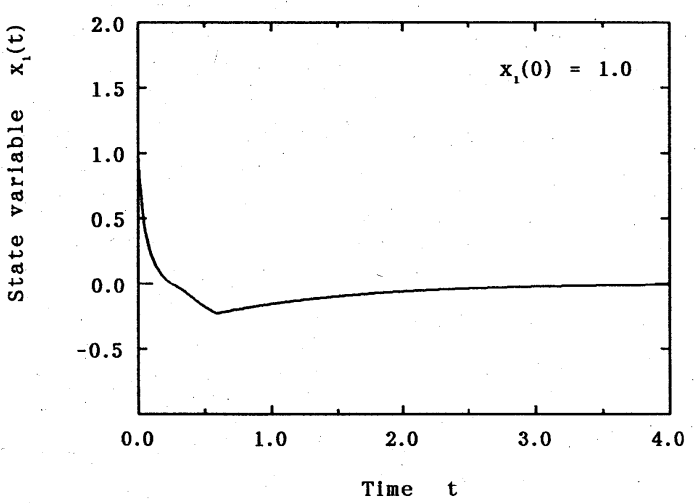

Fig. 1 Response of state variable $x_{1}(t)$ in the numerical example for $x_{1}(0)=1.0$

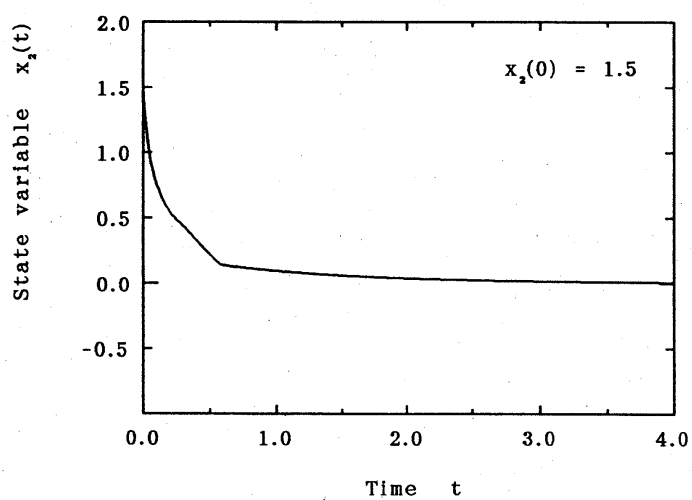

Fig. 2 Response of state variable $x_{2}(t)$ in the numerical example for $x_{2}(0)=1.5$

$$
\delta(x, t)=\frac{x^{T}(t) P x(t)}{\left\|B^{T} P x(t)\right\|^{2}}
$$

and $\rho(x, t)$ satisfies

$$
\|\xi(x, t)\| \leq \rho(x, t)
$$

For simulation, letting

$$
\xi(x, t)=\left[\begin{array}{l}
0.5 \sin \left(x_{1}\right) \\
0.8 \sqrt{\left|x_{1} x_{2}\right|}
\end{array}\right]
$$

then, we may have

$$
\rho(x, t)=\sqrt{0.25 x_{1}^{2}+0.64\left|x_{1} x_{2}\right|}
$$

It is shown from the results of simulation that uncertain closed-loop dynamical system (23), (30) is indeed globally exponentially stable (see Fig. 1 and Fig. 2).

\section{Conclusion}

In this paper, the problem of robust stabilization for a class of dynamical systems with unmatched uncertainties has been discussed. By making use of Lyapunov approach, we have proposed a class of state feedback controllers which can guarantee global exponential stability of this class of uncertain dynamical systems. Furthermore, in terms of the approach developed in this paper, we also obtain an estimate inequality on the rate of convergence.

A numerical example is also given to demonstrate the utilization of the results. It is shown from this numerical example that the approach presented in this paper is effective for a class of dynamical systems with unmatched uncertainties, and may be expected to have some further applications.

\section{Acknowlegments}

The authors would like to thank the anonymous reviewers for their many constructive suggestions and comments that lead to a smoother presentation.

\section{References}

1) S. Gutman: Uncertain Dynamical Systems - A Lyapunov Min-Max Approach, IEEE Trans., AC-24-3, 437/443 (1979)

2) M. J. Corless and G. Leitmann: Continuous State Feedback Guaranteeing Uniform Ultimate Boundedness for Uncertain Dynamic Systems, IEEE Trans., AC -26-5, 1139/1144 (1981)

3) B. R. Barmish, M. J. Corless and G. Leitmann : A New Class of Stabilizing Controllers for Uncertain Dynamical Systems, SIAM Journal on Control and Optimization, 21-2, 246/255 (1983)

4) C. Kravaris and S. Palanki: A Lyapunov Approach for Robust Nonlinear State Feed-back Synthesis, IEEE 
Trans., AC-33-12, 1188/1191 (1988)

5) Y. Liu, M. Cheng and W.B. Gao: A New Control Method and Its Robustness for a Class of Nonlinear Systems, Journal of Optimization Theory and Applications, 59-1, 117/133 (1988)

6) $\mathrm{H}$. Wu and K. Mizukami : Exponential Stability of a Class of Nonlinear Dynamical Systems with Uncertainties, Systems \& Control Letters, 21-4, 307/313 (1993)

7) B. R. Barmish and G. Leitmann: On Ultimate Boundedness Control of Uncertain Systems in the Absence of Matching Assumptions, IEEE Trans., AC-27-1, 153/ 158 (1982)

8) Y. H. Chen and G. Leitmann : Robustness of Uncertain Systems in the Absence of Matching Assumptions, International Journal of Control, 45-5, 1527/1542 (1982)

9) Y. H. Chen : On the Robustness of Mismatched Uncertain Dynamical Systems, ASME Journal of Dynamic Systems, Measurement, and Control, 109-2, 29/35 (1987)

10) Z. Qu: Global Stabilization of Nonlinear Systems with a Class of Unmatched Uncertainties, Systems \& Control Letters, 18-4, 301/307 (1992)

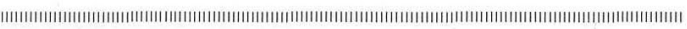

\section{[Authors' Profiles]}

Hansheng WU (Member)

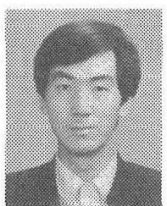

$\mathrm{He}$ received the B.S. and M.S. degrees in automatic control engineering from Northeast University of Technology, P. R. China, in 1982 and 1984, respectively, and $\mathrm{Ph}$. D. degree in information engineering from Hiroshima University, Japan, in 1989. From November 1989 to September 1990, he was a Lecturer of Department of Automatic Control, Northeast University of Technology which has been renamed as Northeastern University since 1993. From October 1990 to March 1992, he was a Postdoctoral Fellow of the University of New South Wales, Australia, in the School of Mechanical and Manufacturing Engineering. From March 1992 to March 1993, he was a Fellow of Postdoctoral Fellowships for Foreign Researchers of Japan Society for the Promotion of Science (JSPS), in Hiro- shima University. Since April 1993, he has joined the Faculty of Integrated Arts and Sciences, Hiroshima University, as an Associate Professor.

His research interests include optimal control, dynamical games, large scale systems, robust control, adaptive control, and the applications of neural networks to control problems.

$\mathrm{Dr}$. Wu is also a member of the Information Processing Society of Japan.

\section{Koichi Mizukami (Member)}

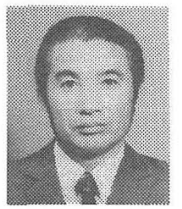

He received the $B$. E. degree in electrical engineering from Hiroshima University in 1958, and M.E. and Doctor of Engineering degrees both in electrical engineering from Kyoto University, Kyoto, Japan in 1960 and 1965, respectively. In April 1963, he joined the Department of Electrical Engineering II at Kyoto University as a research associate. He was a post doctoral fellow of Electrical Engineering at the University of Toronto, Ontario, Canada during 1966 -1968. He then became an associate professor on the Faculty of Engineering at Hiroshima University in October 1968 , and is a professor on the Faculty of Integrated Arts and Sciences at Hiroshima University since April 1977.

His research interests are in the area of control and information sciences, especially functional analysis, optimal control and information theory, fuzzy and neural networks control, information retrieval, and computer graphics. Now, he is a member of the Editorial Boards of the International Journal on Dynamicas and Control, and Journal of Mathematical Analysis \& Applications.

$\mathrm{He}$ is also a member of the IEE of Japan, the Information Processing Society of Japan, and the Institute of Systems, Control and Information Engineers of Japan.

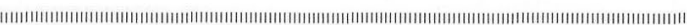

